\title{
Relationship between gut microbiota and heart failure development in an experimental model of hypertensive rats.
}

\author{
Elena Gutiérrez-Calabrés ${ }^{a}$, Adriana Ortega-Hernández, Javier Modrego, Alicia Caro-Vadillo, \\ Dulcenombre Gómez-Garre
}

Hospital Clínico San Carlos - Instituto de Investigación Sanitaria San Carlos (IdISSC). Profesor Martín Lagos s/n, 28040, Madrid.

a. elena.gutierrez@icloud.com

IV Congreso de Señalización Celular, SECUAH 2019.

20-22 de marzo, 2019. Universidad de Alcalá. Alcalá de Henares, Madrid. España

Sesión de paneles.

Keywords: Gut microbiota; heart failure; arterial hypertension; SHR; SHHF

\begin{abstract}
Cardiovascular disease remains the leading cause of death and disability in developed countries. The increase in cardiovascular risk factors, such as hypertension, obesity, diabetes mellitus type II and metabolic syndrome makes it necessary to search for more effective strategies to prevent and modify the course of these cardiometabolic disorders. Heart failure patients show structural and functional intestinal changes due to microcirculatory alterations that can result in a failure of the intestinal barrier function. An altered intestinal mucosal barrier can lead to translocation of microbial products into systemic circulation, aggravating heart failure. The relationship between the alteration of gut microbiota composition, known as dysbiosis, produced metabolites and susceptibility to develop heart failure has highlighted the potential of gut microbiota as a new therapeutic target. The main goal of this study was to compare gut microbiota composition and its association with various cardiac parameters between experimental rat models. To carry out this work, we used Spontaneously Hypertensive Rats (SHR), which rarely develop heart failure; Spontaneously Hypertensive Heart Failure Rats (SHHF) and normotensive rats (WKY) as controls. The taxonomic characterization of gut microbiota, by Next Generation Sequencing of rDNA 16S, demonstrated an alteration of alpha and beta diversity, as well as an alteration of Firmicutes/Bacteroidetes ratio between SHR and SHHF rats throughout the study. The most important changes were observed at 9 months of age before cardiac differences were present. Specifically, a significant decrease in bacteria from genera Oscillibacter, Mucispirillum, Clostridium and Faecalibacterium, related to physiopathological mechanisms associated with heart failure, was observed. Our results demonstrate that gut microbiota dysbiosis present in heart failure animals is prior to the manifestation of cardiac alterations. Therefore, these results suggest that we could use gut microbiota modification as a possible therapeutic strategy to avoid possible cardiac alterations in susceptible individuals to suffer from any cardiovascular pathology.
\end{abstract}

Citation: Gutiérrez-Calabrés, Elena; Ortega-Hernández, Adriana; Modrego, Javier; Caro-Vadillo, Alicia; GómezGarre, Dulcenombre (2019) Relationship between gut microbiota and heart failure development in an

experimental model of hypertensive rats. Proceedings of the IV Congreso de Señalización Celular, SECUAH 2019. 20-22 de marzo, 2019. Universidad de Alcalá. Alcalá de Henares, Madrid. España. Sesión de paneles. dianas 8 (1): e201903p01. ISSN 1886-8746 (electronic) journal.dianas.e201903p01.

URI http://hdl.handle.net/10017/15181

Copyright: (C) Gutiérrez-Calabrés E, Ortega-Hernández A, Modrego J, Caro-Vadillo A, Gómez-Garre D. Some rights reserved. This is an open-access work licensed under a Creative Commons Attribution-NonCommercialNoDerivatives 4.0 International License. http://creativecommons.org/licenses/by-nc-nd/4.0/ 